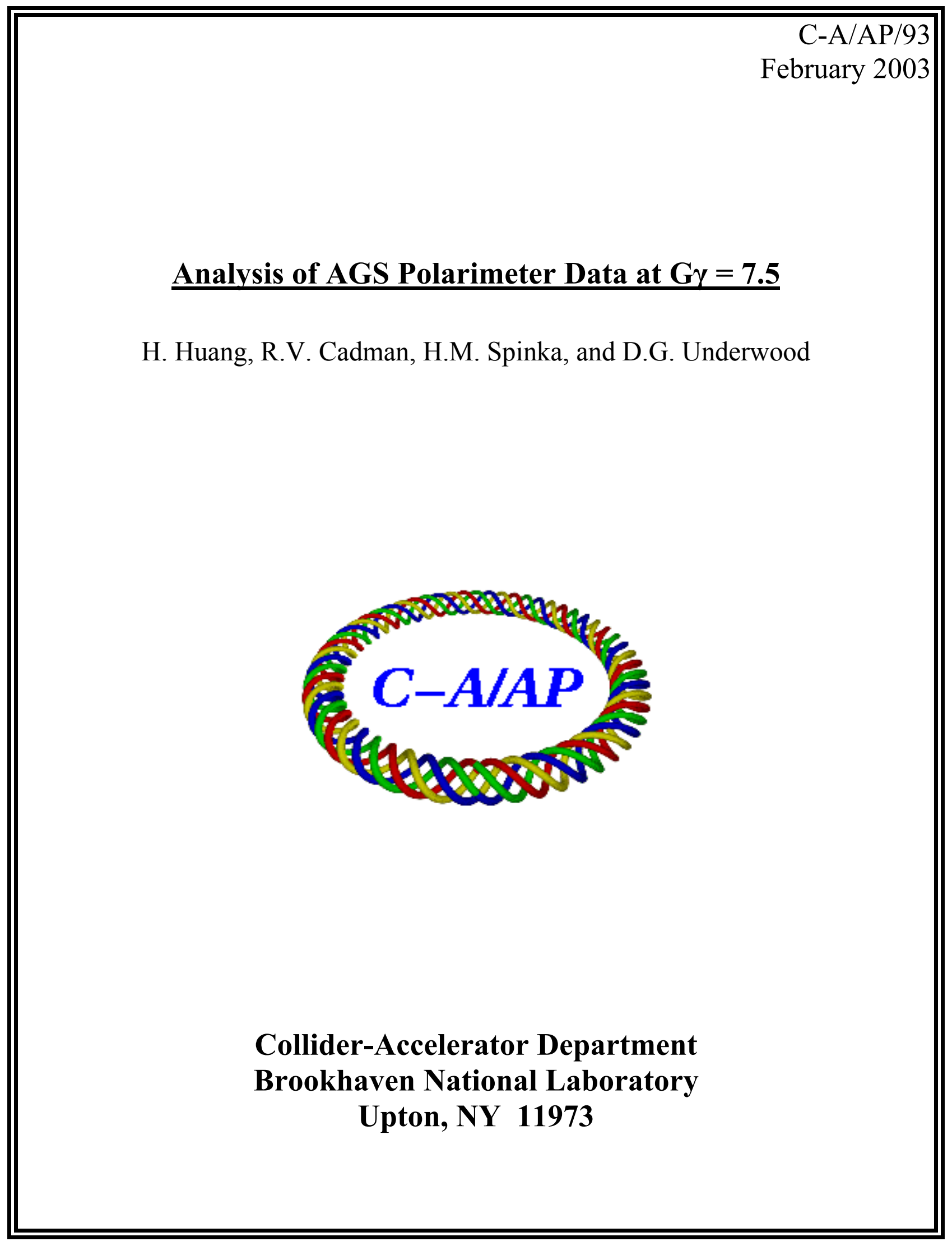




\title{
Analysis of AGS Polarimeter Data at $\mathrm{G} \gamma=7.5$
}

\author{
H. Huang, ${ }^{1}$ R.V. Cadman, ${ }^{2}$ H.M. Spinka, ${ }^{2}$ and D.G. Underwood ${ }^{2}$ \\ ${ }^{1}$ Brookhaven National Laboratory, Upton, New York 11973 \\ ${ }^{2}$ Argonne National Laboratory, Argonne, Illinois 60439
}

(Dated: February 5, 2003)

\begin{abstract}
Data were collected with the AGS internal polarimeter at $G \gamma=7.5$ during the recent FY02 polarized proton run. The addition of new forward scintillation counters permitted an absolute calibration of the polarimeter for both nylon and carbon targets. The results are summarized, and the polarization measured at $G \gamma=7.5$ is compared to that at $200 \mathrm{MeV}$.
\end{abstract}




\section{INTRODUCTION}

The RHIC spin program requires highly-polarized proton beams from the AGS. Extensive experience has demonstrated that it is not easy to maintain the polarization of proton beams as they are accelerated in the AGS. Polarization measurements at various energies in the AGS are needed to guide the accelerator tuning. Until now, the only available polarimeter has been the internal polarimeter designed for E880 which measures the recoil protons from carbon and nylon at large angles. A CNI polarimeter has been installed in the AGS, but it will need to be calibrated using the E880 polarimeter. Clearly the calibration of the E880 polarimeter is crucial in understanding the performance of the AGS with polarized protons.

In September 2000, the E880 polarimeter was run with a carbon target. At $G \gamma=7.5$ $(p=3.81 \mathrm{GeV} / c)$, the measured asymmetries were significantly lower than those measured in earlier years. This might have been caused by depolarization during the acceleration through the Booster and the AGS from $200 \mathrm{MeV}$ to $G \gamma=7.5$. Another explanation is that the carbon target measurements during previous years were contaminated by scattering from a nearby nylon target which was absent from the target ladder in 2000. The feasibility of various explanations has already been discussed in a previous note [1].

To clarify the situation, forward arms were added to the internal polarimeter at angles appropriate to the kinematics of $p p$ elastic scattering. Asymmetries were measured with nylon and carbon targets. To extract the asymmetry for elastic scattering from hydrogen in the nylon, the carbon target was used to subtract the non-hydrogen background in the nylon. The analyzing power for the $p+\mathrm{C}$ reaction can then be calibrated from the previously measured analyzing power for $p+p$.

In this note, the new forward arms and the method for extracting the $p p$ elastic scattering asymmetries are described. Measurements of the analyzing powers for $\vec{p}+\mathrm{C}$ and $\vec{p}+\mathrm{C}_{6} \mathrm{H}_{11} \mathrm{NO}$ are then presented. Finally, previous measurements with the E880 polarimeter are discussed in light of these new results.

\section{APPARATUS}

Forward arms were installed in the AGS internal polarimeter to increase sensitivity to $p p$ elastic scattering. For elastic scattering with a fixed recoil angle, the forward particle 
angle varies with beam energy. At energies higher than $6.5 \mathrm{GeV} / c(G \gamma=12.5)$, the forward particle stays within the beam pipe, and so a forward arm measurement was not performed beyond this energy. Three pairs of detectors were installed, corresponding to $G \gamma=4.7,7.5$, and 12.5. The size of the counters was chosen to match the acceptance of the recoil arms. A sketch of the detector setup is shown in Figure 1. The counters were installed 2.0 meters downstream of the target. A fringe field analysis was done to confirm that there is no strong fringe field from the adjacent dipole magnet at the detector location.

Beam Pipe radius $7.5 \mathrm{~cm}$

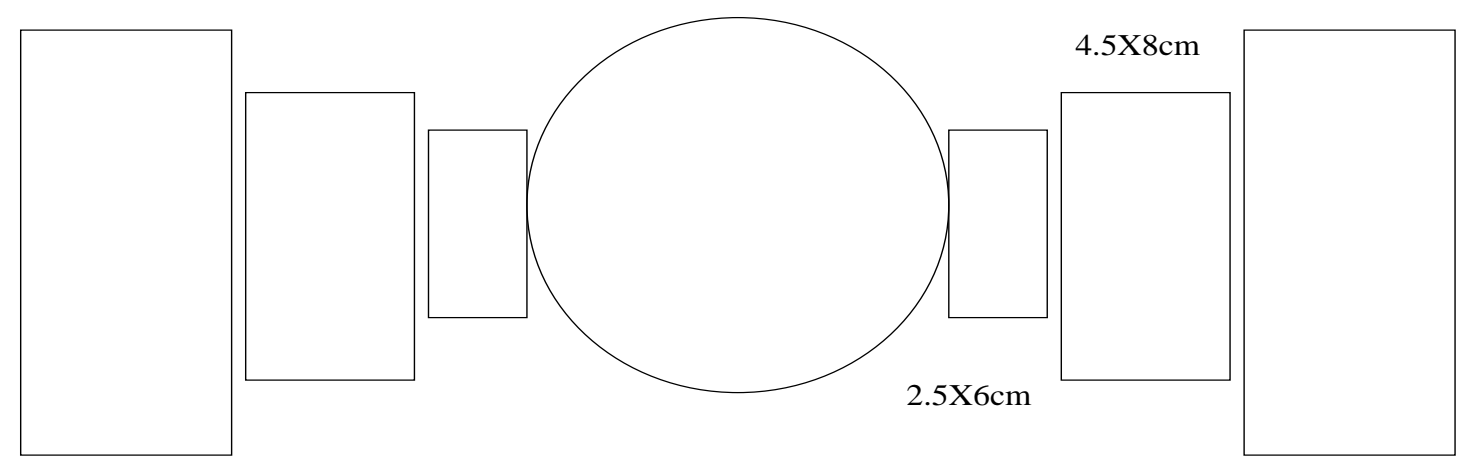

FIG. 1: Sketch of the forward arms. The scintillation counter closest to the beam pipe is for $G \gamma=12.5$ and the counter farthest from the beam pipe is for $G \gamma=4.7$. The middle counter, which was the only one actually used during the run due to the limited available time, is for $G \gamma=7.5$.

The recoil arms were used for the AGS experiment E880. This is the portion of the apparatus which was used to measure the AGS beam polarization in September 2000, and in fact it has not changed since 1995. Each of the recoil arms consists of three scintillators at $27.3 \mathrm{~cm}, 40.0 \mathrm{~cm}$, and $114.0 \mathrm{~cm}$ from the target. Between the second and third scintillators is an adjustable wedge, the position of which is chosen so that for the desired beam energy, the recoil protons will stop in the third scintillator. For the data presented here, the wedge was at the $G \gamma=7.5$ position. The three scintillators in the left arm are identified as $L_{1}, L_{2}$, and $L_{3}$ in order of increasing distance from the target. The corresponding detectors in the right arm are $R_{1}, R_{2}$, and $R_{3}$. The polarimeter is shown in Figure 26 of Ref. [2]. The other detectors shown in that figure were not used in the present work. 
One nylon target and two carbon targets were mounted on the target ladder. The carbon targets were separated by $2 \mathrm{~cm}$, and the nylon target was more than $1 \mathrm{~cm}$ from the nearer carbon target as shown in Figure 2. Carbon asymmetries were measured with the carbon target which was farther from the nylon target. That target contained seven fibers, each $8 \mu \mathrm{m}$ in diameter; these were counted under a microscope after the experiment. The nylon target was $102 \mu \mathrm{m}$ in diameter. The backup carbon target could only be used if the nylon target was not present. The number of fibers in this target was not counted, but it is assumed to be identical to the primary carbon target.

Most of the calibration runs were done on Nov. 16, 2001 between noon and midnight. The beam intensity was $\sim 5 \times 10^{9}$ protons per AGS bunch. A full range target scan showed clearly that the two targets used for the calibration runs were well separated, as shown in Figure 2. In the figure, the electronic OR of coincidences in the first two scintillators in each recoil arm (i.e., $\left.\left(L_{1} \cdot L_{2}\right) \oplus\left(R_{1} \cdot R_{2}\right)\right)$ is plotted as a function of target position. The angular and momentum acceptance of the first two counters is larger than that of the full recoil arm. This provides a better matching of $p p$ elastic to $p \mathrm{C}$ quasi-elastic scattering, and thus a better luminosity normalization. In addition, the data are fit to three Gaussians as shown in the figure. Because the width of each Gaussian is entirely due to the beam size, the three Gaussians are constrained to have equal widths in the fit. See Ref. [2] for details of the electronics.

During the calibration runs, the nylon target actually killed the beam completely in about $0.5 \mathrm{sec}$, so most of the time at flattop there were no coincidences. There were counts with the carbon target for nearly the full 1.2 sec flattop, from the time the target was inserted until the target was removed. It was estimated that the nylon target peak would be 2 to 4 times higher if a correction were made for integrated beam current in the different target positions. Furthermore, there was a problem with an electronics module (Jorway 101), and the gate to record events was not applied to the NIM bin containing the counter electronics. This may explain why the peaks in Figure 2 are so broad, because events were recorded while the targets were moving into the beam, as well as while they were in the final positions. These two effects are also apparent from the fits to the target scan results, where the carbon target data near position 1400 are significantly narrower than the fit.

The relative sizes of the nylon and carbon target peaks are consistent with the known linear densities of the targets. With the correction estimated above, the luminosity from 


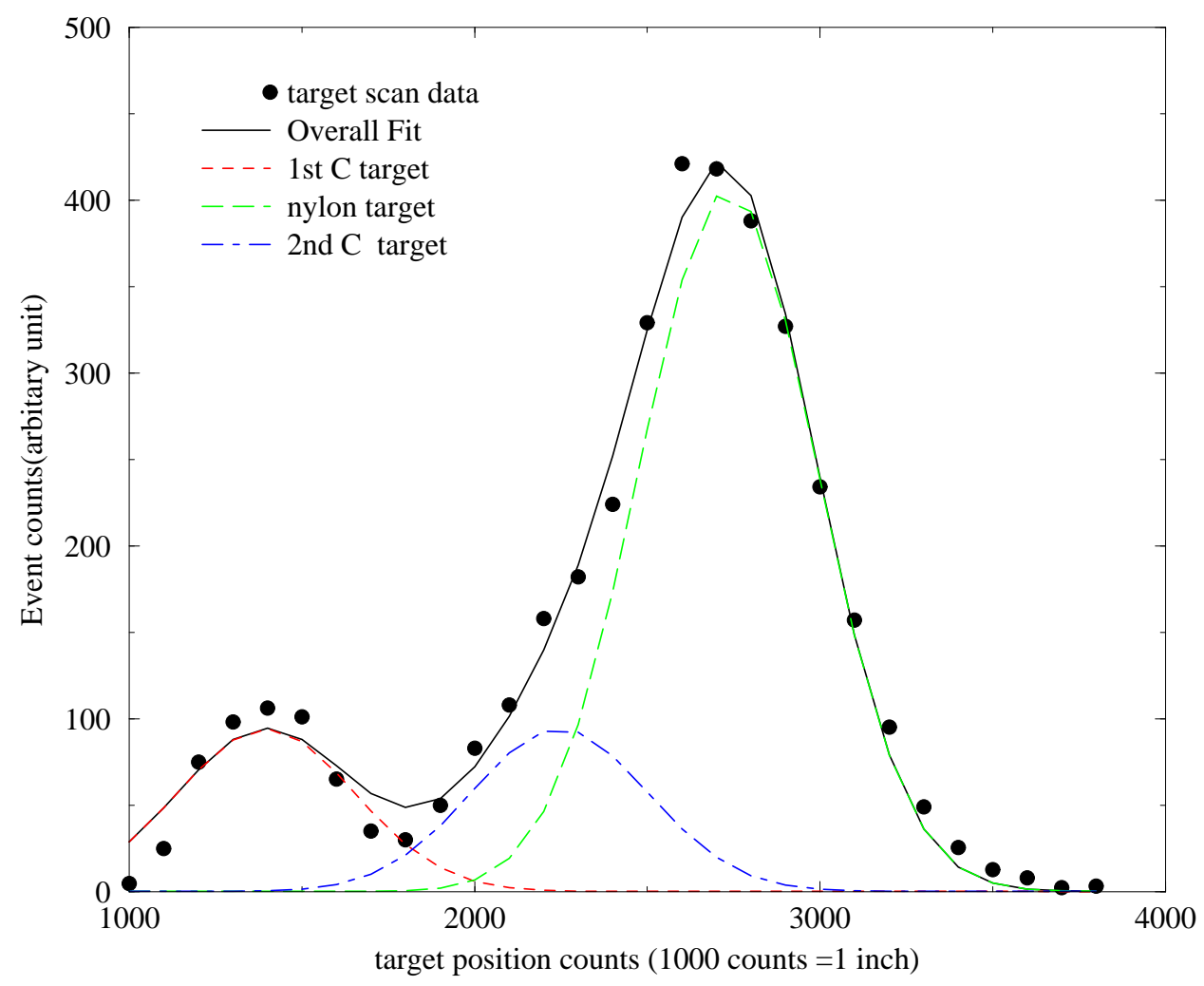

FIG. 2: Target scan at $G \gamma=7.5$. The horizontal axis is the target position measured in control counts, where 1000 counts is about one inch. The vertical axis is the relative number of events in the recoil arms.

the nylon target is 8 to 16 times larger than that of the carbon target. For comparison, the target densities are $107.6 \mu \mathrm{g} / \mathrm{cm}$ for nylon and $1.19 \mu \mathrm{g} / \mathrm{cm}$ for each carbon fiber. With seven fibers, the ratio of nylon to carbon is 12.9 in agreement with the estimated luminosities.

The carbon target runs were taken with target position 1400, and nylon target runs were taken with target positions 3000 and 3200. The scan shows the target separation was around 1400 counts during the most recent run, while it had been around 1000 counts in previous years. Contamination of the carbon asymmetry data in 1997 and 1999 was further compounded by the larger horizontal emittance of $40 \pi \mathrm{mm}$-mrad in those years compared to $20 \pi \mathrm{mm}$-mrad for the present run.

The measurements described below were taken under the same conditions as above with two exceptions. First, a signal in either recoil arm required a three-fold coincidence, e.g. $L_{1} \cdot L_{2} \cdot L_{3}$ for the left arm. Second, the DAQ gate was successfully applied to veto events 
occurring while the target was moving into and out of the beam. For three runs (4034, 4036, and 4039) this gate was $1.2 \mathrm{sec}$ wide; for all other measurements it was $0.7 \mathrm{sec}$ wide.

\section{BACKGROUND SUBTRACTION}

An upper limit to the background contribution can be estimated from the observed event rates for the carbon and nylon targets both for the recoil arms $(R)$ alone and for the forward-recoil coincidences (FR). This estimate assumes that the cross sections per nucleon for inclusive proton scattering into the recoil arms are roughly equal for all nuclei in nylon. Defining the number of events for the nylon (fishline), carbon, hydrogen, and background (non-hydrogen in nylon) to be $N_{F}, N_{C}, N_{H}$, and $N_{B}$, respectively, then the number of forward-recoil coincidences can be written as

$$
\begin{aligned}
& \left(N_{F}\right)_{F R}=\left[(1-p) d \sigma_{C}+p d \sigma_{H}\right] \cdot I_{F} \\
& \left(N_{C}\right)_{F R}=d \sigma_{C} \cdot I_{C} \\
& \left(N_{B}\right)_{F R}=(1-p) d \sigma_{C} \cdot I_{F}=\left(N_{F}\right)_{F R}-\left(N_{H}\right)_{F R} \\
& \left(N_{H}\right)_{F R}=p d \sigma_{H} \cdot I_{F} .
\end{aligned}
$$

In these expressions, $I_{F}$ and $I_{C}$ are integrals of the beam current on the nylon (fishline) and carbon targets, taking into account the beam spot shape, target linear density, beam lifetime, etc. The effective cross sections per nucleon for forward-recoil coincidences, weighted by acceptance of the detectors, are $d \sigma_{H}$ and $d \sigma_{C}$. The percentage by weight of hydrogen in the nylon $\left(\mathrm{C}_{6} \mathrm{H}_{11} \mathrm{NO}\right)$ target is $p \cong 0.097$. The background fraction is

$$
\begin{aligned}
r & =\left(N_{B}\right)_{F R} /\left(N_{F}\right)_{F R} \\
& =\frac{(1-p) d \sigma_{C}}{(1-p) d \sigma_{C}+p d \sigma_{H}} \leq 1
\end{aligned}
$$

To obtain similar expressions for the recoil arms alone, one makes the substitution

$$
\begin{aligned}
& d \sigma_{C} \longrightarrow A d \sigma_{C} \\
& d \sigma_{H} \longrightarrow B d \sigma_{H}
\end{aligned}
$$


and the number of events is then given by

$$
\begin{aligned}
& \left(N_{F}\right)_{R}=\left[(1-p) A d \sigma_{C}+p B d \sigma_{H}\right] \cdot I_{F} \\
& \left(N_{C}\right)_{R}=A d \sigma_{C} \cdot I_{C} \\
& \left(N_{B}\right)_{R}=(1-p) A d \sigma_{C} \cdot I_{F}=\left(N_{F}\right)_{R}-\left(N_{H}\right)_{R} \\
& \left(N_{H}\right)_{R}=p B d \sigma_{H} \cdot I_{F} .
\end{aligned}
$$

In this case, the cross sections per nucleon for carbon and hydrogen are expected to be similar:

$$
A d \sigma_{C} \approx B d \sigma_{H}
$$

Consider the ratio of ratios,

$$
\begin{aligned}
R & =\frac{\left(N_{C}\right)_{F R} /\left(N_{C}\right)_{R}}{\left(N_{F}\right)_{F R} /\left(N_{F}\right)_{R}} \\
& =\frac{d \sigma_{C} / A d \sigma_{C}}{\left[(1-p) d \sigma_{C}+p d \sigma_{H}\right] /\left[(1-p) A d \sigma_{C}+p B d \sigma_{H}\right]} \\
& =\frac{(1-p) d \sigma_{C}+(B / A) p d \sigma_{H}}{(1-p) d \sigma_{C}+p d \sigma_{H}} \\
& =r \cdot\left[1+(B / A) \frac{p d \sigma_{H}}{(1-p) d \sigma_{C}}\right] .
\end{aligned}
$$

Thus $R$ is a measurable upper limit to the desired background ratio $r$. Furthermore, the term $(B / A) \frac{p d \sigma_{H}}{(1-p) d \sigma_{C}}$ is small compared to one because of Eq. (5) and

$$
p \ll(1-p) .
$$

Therefore, it is expected that $R$ is only slightly larger than $r$, permitting a good estimate of the background fraction. The ratio $R$ was computed from a set of runs taken at $G \gamma=7.5$ at nearly the same time as the target scan in Figure 2. The result is

$$
R=0.152 \pm 0.021
$$

The adopted background ratio is slightly smaller (taking into account Eqs. (5) and (6)):

$$
r=0.137 \pm 0.019
$$

This uncertainty is entirely due to the statistical uncertainty in $R$. The systematic error due to our assumption of equal cross sections per nucleon is negligible; if we assigned an error $\left(B d \sigma_{H}\right) /\left(A d \sigma_{C}\right)=1.0 \pm 0.5$, the result would change to $r=0.137 \pm 0.020$. 


\section{ASYMMETRIES}

The beam polarization was calculated using the known analyzing power for $p p$ elastic scattering and the measured forward-recoil asymmetry for the nylon target. The carbon target was used to subtract the non-hydrogen background in the nylon. This beam polarization was then used to calculate the analyzing powers of the recoil arms for both nylon and carbon. The data used for this analysis are listed in the Tables I and II.

\begin{tabular}{|c|c|c|c|}
\hline $\begin{array}{c}\text { Run } \\
\text { number }\end{array}$ & $\begin{array}{c}\text { Target } \\
\text { position }\end{array}$ & $\begin{array}{c}\text { RECOIL } \\
\text { asymmetry }\left(\times 10^{-3}\right)\end{array}$ & $\begin{array}{c}\text { FORWARD*RECOIL } \\
\text { asymmetry }\left(\times 10^{-3}\right)\end{array}$ \\
\hline 4034 & 1400 & $30.6 \pm 1.0$ & $67.1 \pm 3.9$ \\
\hline 4039 & 1400 & $31.3 \pm 1.3$ & $70.2 \pm 5.3$ \\
\hline 4040 & 1400 & $33.3 \pm 1.1$ & $65.9 \pm 4.2$ \\
\hline 4055 & 1400 & $29.4 \pm 1.3$ & $58.0 \pm 5.3$ \\
\hline 4057 & 1400 & $30.4 \pm 1.2$ & $69.2 \pm 5.4$ \\
\hline
\end{tabular}

TABLE I: Magnitudes of asymmetries observed with the carbon target. The averages are (31.06 \pm $0.52) \times 10^{-3}\left(\chi^{2} /\right.$ d.o.f. $\left.=1.18\right)$ for the recoil arms and $(66.18 \pm 2.10) \times 10^{-3}\left(\chi^{2} /\right.$ d.o.f. $\left.=0.91\right)$ for coincidences of the recoil and forward arms.

The asymmetry from the nylon can be expressed in terms of the asymmetry of its constituents:

$$
\epsilon_{F}=\epsilon_{C} \times r+\epsilon_{H} \times(1-r),
$$

where $r=0.137 \pm 0.019$ is the background ratio, and $\epsilon_{C}$ and $\epsilon_{F}$ are the asymmetries in the coincidence of forward and recoil arms measured for carbon and nylon targets, respectively. The $p p$ elastic analyzing power is $A_{N}=0.1863 \pm 0.0112$ from the empirical fit in Ref. [3]. The beam polarization is $P=A_{N} / \epsilon_{H}$.

Using the average asymmetries of forward and recoil coincidences measured with carbon and nylon one obtains

$$
\epsilon_{H}=0.1268 \pm 0.0012 \pm 0.0013
$$

Here the first error is statistical and the second is the systematic error due to the error in $r$. The uncertainty due to the assumption that carbon, oxygen, and nitrogen contribute equal 


\begin{tabular}{|c|c|c|c|}
\hline $\begin{array}{c}\text { Run } \\
\text { number }\end{array}$ & $\begin{array}{c}\text { Target } \\
\text { position }\end{array}$ & $\begin{array}{c}\text { RECOIL } \\
\text { asymmetry }\left(\times 10^{-3}\right)\end{array}$ & $\begin{array}{c}\text { FORWARD*RECOIL } \\
\text { asymmetry }\left(\times 10^{-3}\right)\end{array}$ \\
\hline 4036 & 3000 & $73.8 \pm 1.0$ & $118 \pm 1.9$ \\
\hline 4043 & 3200 & $74.7 \pm 3.5$ & $125 \pm 8.2$ \\
\hline 4047 & 3000 & $73.8 \pm 1.7$ & $123 \pm 3.2$ \\
\hline 4048 & 3100 & $71.2 \pm 2.0$ & $110 \pm 4.1$ \\
\hline 4051 & 3200 & $70.9 \pm 2.1$ & $120 \pm 4.9$ \\
\hline 4053 & 3000 & $71.9 \pm 1.3$ & $119 \pm 2.5$ \\
\hline 4056 & 3000 & $70.4 \pm 1.8$ & $117 \pm 3.3$ \\
\hline 4058 & 3000 & $73.3 \pm 1.1$ & $119 \pm 2.1$ \\
\hline
\end{tabular}

TABLE II: Magnitudes of asymmetries from the nylon target. The averages are $(72.74 \pm 0.52) \times 10^{-3}$ $\left(\chi^{2} /\right.$ d.o.f. $\left.=0.89\right)$ for the recoil arms and $(118.51 \pm 1.02) \times 10^{-3}\left(\chi^{2} /\right.$ d.o.f. $\left.=1.03\right)$ for coincidences of the recoil and forward arms.

asymmetries is not included. To our knowledge there is no data on oxygen or nitrogen to support or refute this assumption near the relevant energy. If we applied a $100 \%$ error to the asymmetries due to nitrogen and oxygen, i.e. $\epsilon_{N O}=0.066 \pm 0.066$, the error in $\epsilon_{H}$ would increase to $3 \%$. This error, which is certainly too large, is less than the $6 \%$ uncertainty in $A_{N}$ and therefore is ignored. The resulting polarization is

$$
P=0.681 \pm 0.006 \pm 0.041
$$

Here the systematic error (the second error) is dominated by the uncertainty in $A_{N}$. Using this derived polarization and the recoil arm asymmetries, the analyzing powers of the recoil arms can be computed:

$$
\begin{gathered}
A_{N}^{C}=(45.6 \pm 0.8 \pm 0.4 \pm 2.8) \times 10^{-3}, \\
A_{N}^{F}=(106.9 \pm 0.8 \pm 1.0 \pm 6.5) \times 10^{-3} .
\end{gathered}
$$

Here the contributions to the overall error are shown separately. The three errors, in order, are due to the statistical error in the asymmetry measurement, the statistical error in the polarization, and the systematic error in the polarization. Only the first error is uncorrelated between the two measurements. 
Given the measured asymmetries, one can express the polarization in terms of the assumed value for the background ratio $r$. Table III shows how different background ratios would effect the values of the polarization and $A_{N}^{C}$. As indicated, if the background ratio changes by $\pm 0.012, A_{N}^{C}$ will vary by $\pm 0.3 \times 10^{-3}$. The variation in $A_{N}^{C}$ caused by the uncertainty in the background ratio is included in the quoted error on $A_{N}^{C}$.

\begin{tabular}{|c|c|c|}
\hline Background ratio & Polarization & $A_{N}^{C}\left(\times 10^{-3}\right)$ \\
\hline 0.05 & 0.651 & 47.72 \\
\hline 0.09 & 0.664 & 46.78 \\
\hline 0.125 & 0.676 & 45.93 \\
\hline 0.137 & 0.681 & 45.63 \\
\hline 0.15 & 0.686 & 45.30 \\
\hline 0.16 & 0.690 & 45.03 \\
\hline 0.25 & 0.730 & 42.56 \\
\hline
\end{tabular}

TABLE III: Beam polarization and effective p-Carbon inclusive analyzing power for the recoil arms. Various background ratios are assumed. The actual beam polarization and effective pCarbon inclusive analyzing power are only weakly dependent on the background ratio.

\section{DISCUSSION}

The forward arms turned out to be very useful in determining the analyzing power for the AGS polarimeter at low energy $(G \gamma=7.5)$. The polarization extracted from these measurements is in agreement with the source polarization $\sim 70 \%$. The consistency of these independent measurements is an indication that the polarization is better understood. There were several runs with the carbon target for $G \gamma=7.5$ in addition to the calibration measurement described above. In Table IV, these measurements are listed and compared to measurements with the $200 \mathrm{MeV}$ polarimeter. These data are also plotted in Figure 3. Only the statistical uncertainties in the measurements with the $200 \mathrm{MeV}$ polarimeter are quoted; the systematic error may be $3 \%$ or more, and will be discussed in another note [4]. It seems that the $200 \mathrm{MeV}$ polarimeter detectors were still saturated with higher current $(\sim 200 \mu \mathrm{A})$, so the beam intensity was decreased to allow polarization measurements at $200 \mathrm{MeV}$. 


\begin{tabular}{|c|c|c|c|c|c|c|c|}
\hline $\begin{array}{c}\text { run } \\
\text { number }\end{array}$ & $\begin{array}{c}\text { asymmetry } \\
\left(\times 10^{-3}\right)\end{array}$ & $\begin{array}{c}G \gamma=7.5 \\
\text { polarization }\end{array}$ & $\begin{array}{c}200 \mathrm{MeV} \\
\text { polarization }\end{array}$ & $\begin{array}{c}200 \mathrm{MeV} \\
\text { current }(\mu \mathrm{A})\end{array}$ & $\begin{array}{c}\text { target at } \\
200 \mathrm{MeV}\end{array}$ & date & time \\
\hline $4034-50$ & $31.06 \pm 0.52$ & $68.1 \pm 1.0$ & $67.8 \pm 2.6$ & 100 & $\mathrm{C}$ & $11 / 16 / 01$ & \\
\hline 4284 & $35.83 \pm 0.87$ & $78.5 \pm 2.6$ & $73.0 \pm 1.3$ & 100 & $\mathrm{C}$ & $12 / 8 / 01$ & $12: 15$ \\
\hline 4285 & $35.13 \pm 0.70$ & $77.0 \pm 2.3$ & $72.5 \pm 1.0$ & 100 & $\mathrm{C}$ & $12 / 8 / 01$ & $12: 32$ \\
\hline 4286 & $35.27 \pm 0.62$ & $77.3 \pm 2.2$ & $73.6 \pm 1.3$ & 50 & $\mathrm{C}$ & $12 / 8 / 01$ & $12: 46$ \\
\hline 4959 & $32.85 \pm 0.82$ & $72.0 \pm 2.4$ & $71.2 \pm 0.9$ & 100 & $\mathrm{C}$ & $1 / 5 / 02$ & $17: 36$ \\
\hline 4969 & $33.68 \pm 0.64$ & $73.8 \pm 2.1$ & $73.2 \pm 0.3$ & 100 & $\mathrm{CD}_{2}$ & $1 / 6 / 02$ & $01: 13$ \\
\hline 4970 & $34.26 \pm 0.69$ & $75.1 \pm 2.2$ & $73.2 \pm 0.3$ & 100 & $\mathrm{CD}_{2}$ & $1 / 6 / 02$ & $01: 19$ \\
\hline 4995 & $32.79 \pm 0.70$ & $71.9 \pm 2.2$ & $72.3 \pm 0.3$ & 100 & $\mathrm{CD}_{2}$ & $1 / 6 / 02$ & $23: 30$ \\
\hline 5452 & $32.90 \pm 1.40$ & $72.1 \pm 3.5$ & $70.0 \pm 1.6$ & 60 & $\mathrm{C}_{1}$ & $1 / 23 / 02$ & $18: 26$ \\
\hline
\end{tabular}

TABLE IV: Comparison of polarization measured at $200 \mathrm{MeV}$ and at $\mathrm{G} \gamma=7.5$. For the measurements in the AGS, the quoted uncertainty does not include the $6 \%$ normalization uncertainty due to $A_{N}$, and the other uncertainties are added in quadrature. The quoted errors in the polarization at $200 \mathrm{MeV}$ are statistical only.

The average ratio of AGS polarization measurements to the $200 \mathrm{MeV}$ polarization measurements is $1.025 \pm 0.011$ with $\chi^{2} /$ d.o.f $=0.81$. The ratio is consistent with one although the AGS measurements were systematically higher. If runs 4284-4286 are excluded, the ratio is $1.010 \pm 0.013$.

A previous analysis [1] evaluated the possibility that the larger carbon asymmetries during earlier runs were due to scattering from the nearby nylon target. In fact the carbon asymmetry measured in July 1997 is greater than the carbon analyzing power derived in Equation (13). This fact strongly supports the hypothesis of contamination from the nylon.

The asymmetries measured in September 2000 remain a puzzle. They are about 30\% lower than the measurements presented here, while the source polarization was not more than 15\% lower. Four possible problems with the 2000 run might explain this discrepancy: lower (or not stable) source polarization, polarization loss in the AGS before $G \gamma=7.5$ (if the Snake was not strong enough), a malfunction of the AGS polarimeter in the FY00 run, and polarization loss in the Booster. The last three possibilities were studied at the end of the FY00 run and it was believed that they could be ruled out. Regarding the question 


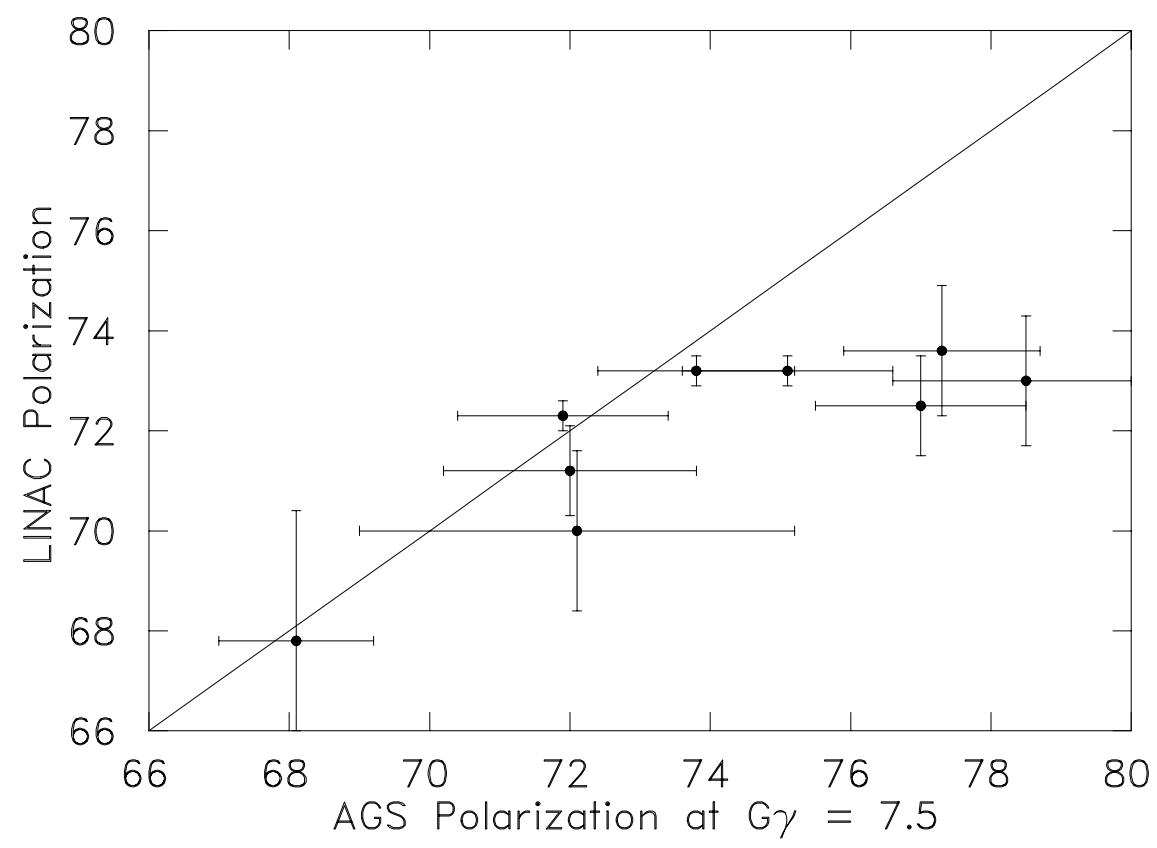

FIG. 3: Comparison of polarization measured at $200 \mathrm{MeV}$ and at $\mathrm{G} \gamma=7.5$. The horizontal error bars indicate only the statistical error in the corresponding asymmetry measurement, because that is the only independent error between the data points. The exception is the point farthest to the left, which corresponds to the initial measurement to which all other points are calibrated. In this case the error bar indicates the systematic error which is common to the other points but which is actually not an error in the measurement of the calibration point; in other words it indicates the amount by which the other points could vary in unison with respect to the calibration point. The vertical error bars indicate statistical errors only.

of the source polarization, it should be noted that the polarization at the end of LINAC (200 MeV) was not measured constantly during the FY00 run. In fact, it was only measured a few times over the two weeks running time, and only with a lower source current. At the time, the polarimeter detectors were saturated with the regular beam current of $100 \mu \mathrm{A}$, and so the polarimeter was used with only $10 \mu \mathrm{A}$ of beam. The beam polarization may have fluctuated from time to time during the FY00 run, and in fact the source polarization might have increased when the beam current was decreased.

Figure 4 is re-plotted from Ref. [1] and includes the most recent data point. The arrows around the September 2000 and July 1996 data indicate that there were no constraints on $A_{N}^{F} / A_{N}^{C}$ in both years and there was only a lower limit on $A_{N}^{C}$ from the July 1996 run. 
Including the November 2001 data, all data are in agreement except the data point of September 2000.

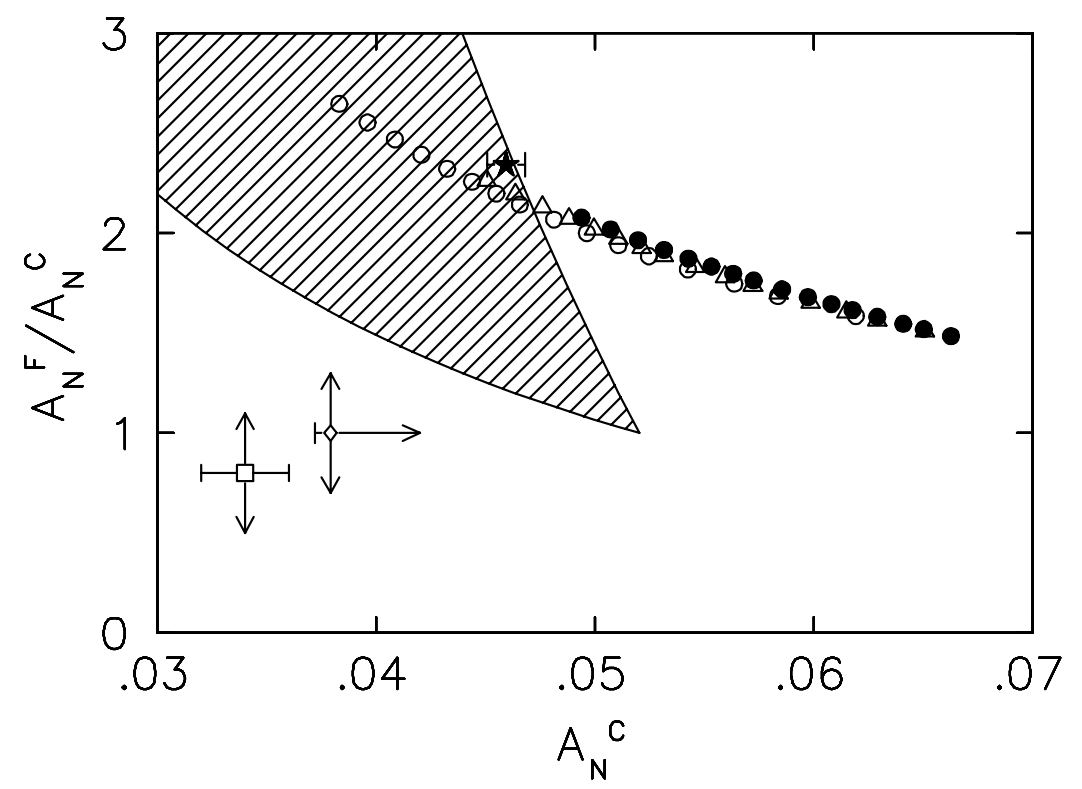

FIG. 4: Results of the analysis of five run periods at $G \gamma=7.5$. The data for Sept. 2000 ( $\square$ ) and July $1996(\diamond)$ provide values for $A_{N}^{C}$ but not for $A_{N}^{F}$. With a range of possible beam sizes $7.5 \mathrm{~mm} \leq \sigma \leq 12.5 \mathrm{~mm}$, the March 1999 results limit $A_{N}^{F}$ and $A_{N}^{C}$ to the region marked off by diagonal lines. The July 1997 data $(\bullet)$ give a narrow band of values, where the full range of allowable beam size, from $\sigma=7.5$ to $12.5 \mathrm{~mm}$, is shown. Also shown are values allowed by the July 1997 data when an offset of the beam from the target is assumed (o, $5.0 \mathrm{~mm}$, and $\triangle, 2.5 \mathrm{~mm}$ ). Since the November 2001 measurements have no uncertainties due to beam size or offsets, only a single point is shown $(\star)$.

Another puzzle is the Saclay measurement of $A_{N}^{C}=(33 \pm 0.2) \times 10^{-3}$ at $p=3.62 \mathrm{GeV} / c[5]$, which is very different from our result. These two measurements are at approximately identical $t$, however, there are some detailed differences between the Saclay and AGS polarimeters, in addition to the $0.19 \mathrm{GeV} / c$ momentum difference. For example, the angles are slightly different $\left(73^{\circ}\right.$ vs. $\left.77^{\circ}\right)$, and the angular acceptances, energy losses, and degrader used in the AGS polarimeter are also different. Note that at $p=3.20 \mathrm{GeV} / c$ the Saclay result was substantially larger $\left(A_{N}^{C}=0.045 \pm 0.003\right)$, which may indicate a flawed measurement at $p=3.62 \mathrm{GeV} / c$. 


\section{CONCLUSIONS}

The analyzing powers of the AGS polarimeter for both carbon and nylon targets have been measured at $G \gamma=7.5$. Forward arms were installed in the polarimeter to allow this calibration from the well-known analyzing power for $p p$ elastic scattering at this energy. The data indicate that previous carbon target asymmetries were contaminated by the nearby nylon target, but the low asymmetries measured in September 2000 are still not understood.

We plan to repeat this measurement during the FY2003 RHIC run. This calibration is important because knowledge of the polarization in the AGS is critical to optimize its performance as a source of polarized protons for RHIC and because it will be used to calibrate the AGS CNI polarimeter which is now being installed. Given the discrepancies between 2000 and 2001, another measurement would be prudent.

\section{ACKNOWLEDGMENT}

The authors would like to thank Dr. W. Meng for a calculation of the fringe field near AGS dipole magnets.

[1] R. Cadman et al., "Analysis of Low Energy AGS Polarimeter Data and Potential Consequence for RHIC Spin Physics," C-A/AP/56 (May 2001).

[2] C.E. Allgower et al., Phys. Rev. D 65, 092008 (2002).

[3] H. Spinka et al., Nucl. Instrum. Methods, 211, 239 (1983).

[4] A. Bravar et al., "Analysis of Polarimeter Data for the 2001-2002 RHIC Run," C-A Note (in preparation).

[5] J. Bystricky et al., Let. Nuo. Cim. 41, 285 (1984). 